\title{
Same-day cancellation of cardiac surgery: A retrospective review at a large academic tertiary referral center
}

\author{
Mark M. Smith, MD, ${ }^{\mathrm{a}}$ William J. Mauermann, MD, ${ }^{\mathrm{a}}$ David J. Cook, MD, ${ }^{\mathrm{a}, \mathrm{b}}$ Joseph A. Hyder, MD, PhD, ${ }^{\mathrm{a}, \mathrm{c}}$ \\ Joseph A. Dearani, MD, ${ }^{d}$ and David W. Barbara, MD $^{\mathrm{a}, \mathrm{c}}$
}

Background: Same-day cancellations of cardiac surgery are unfortunate and costly occurrences that potentially place patients at risk of adverse events.

Methods: We retrospectively reviewed all same-day cancellations of cardiac operations requiring cardiopulmonary bypass from 2010 to 2012 at a large academic tertiary referral center.

\begin{abstract}
Results: Of 7081 cardiac operations, 134 patients experienced 142 same-day cancellations of cardiac surgery. The foreseeable cancellation causes comprised $17 \%$ of cancellations, nonforeseeable $59 \%$, and indeterminate $24 \%$. The reasons for cancellation were medical (51\%), administrative or scheduling (17\%), unknown (12\%), procedure no longer required (11\%), patient cancellation $(6 \%)$, and preincisional complication ( $3 \%)$. The mean interval from the patient seeing a nonsurgeon provider to cancellation was $8.6 \pm 16.2$ days. No statistically significant differences existed between the foreseeable and nonforeseeable cancellations and the timing of the visit to a nonsurgeon provider $(15,30,45$, and 60 days) before cancellation. Seven patients $(5 \%)$ had died within 30 days of cancellation. Of those subsequently operated on, the mean interval from cancellation to performance of the operation was $12.2 \pm 22.7$ days. Of the 142 cancelled surgical procedures, $28(20 \%)$ were never subsequently performed.
\end{abstract}

Conclusions: Same-day cancellation of cardiac surgery occurred infrequently ( $2 \%$ of cardiac operations performed) at our institution. The cancellations were for foreseeable causes in a few cases. Seeing a nonsurgeon provider more recently before cancellation was not significantly associated with nonforeseeable versus foreseeable cancellations. Although uncommon at our institution, same-day cancellations should be viewed as an opportunity for practice improvement, given the foreseeable nature of some cancelations, associated 30-day mortality, and portion of patients not subsequently undergoing cardiac surgery. (J Thorac Cardiovasc Surg 2014;148:721-5)

Same-day cancellation of cardiac surgery is an unfortunate occurrence with financial and patient satisfaction implications. ${ }^{1,2}$ Patients undergoing cardiac surgery often have conditions that are incapacitating or even life-threatening, for which cancellation or delay of the operation will place them at risk of adverse outcomes. Previous studies evaluating cancellations among various surgical subspecialties have reported cancellation rates of $15 \%$ to $24 \%$ for cardiothoracic surgical procedures, with incomplete medical workup, a lack of postoperative beds, and administrative matters as common reasons. ${ }^{1,3-5}$ Only 1 previous study specifically evaluated same-day cancellations of cardiac

From the Department of Anesthesiology, ${ }^{\mathrm{a}}$ Center for the Science of Health Care Delivery, ${ }^{\mathrm{b}}$ Department of Critical Care Medicine, ${ }^{\mathrm{c}}$ and Division of Cardiovascular Surgery, ${ }^{\mathrm{d}}$ Department of Surgery, Mayo Clinic College of Medicine, Rochester, Minn.

Disclosures: Authors have nothing to disclose with regard to commercial support.

Received for publication Oct 28, 2013; revisions received Feb 27, 2014; accepted for publication March 3, 2014; available ahead of print April 1, 2014.

Address for reprints: David W. Barbara, MD, Departments of Anesthesiology and Critical Care Medicine, Mayo Clinic College of Medicine, 200 First St, SW,

Rochester, MN 55905 (E-mail: Barbara.David@mayo.edu).

$0022-5223 / \$ 36.00$

Copyright (c) 2014 by The American Association for Thoracic Surgery

http://dx.doi.org/10.1016/j.jtcvs.2014.03.002 surgery. ${ }^{3}$ To our knowledge, studies specifically examining same-day cancellation of cardiac surgery in developed countries have not been published. The purpose of our investigation was to retrospectively examine same-day cancellation of cardiac operations at a large tertiary academic referral center, with a particular interest toward determining the cancellation rate; identifying the incidence and causes of foreseeable cancellations; and quantifying the operative delay.

\section{METHODS}

The Mayo Foundation institutional review board approved the present study, and all patients provided written informed consent for the use of their records for research purposes. A computerized search of the medical records database from January 1, 2010, through December 31, 2012, was conducted to identify patients scheduled for cardiac surgery who had had a cancellation on the day of the planned operation. Only cardiac operations requiring cardiopulmonary bypass were included. Patients were excluded from analysis if the cancellation was for a planned operating room hold for percutaneous valve surgery performed outside the main surgical suite, for a procedure not listed for cardiopulmonary bypass (eg, extracorporeal membranous oxygenation weaning, mediastinal washout, pacemaker and/or lead extraction), or for age $<18$ years at cancellation.

All available electronic records were reviewed by 1 of us (M.M.S.). The following information was recorded in a database: demographic data, date of cancellation, location of cancellation (preoperative or intraoperative), 


\section{Abbreviation and Acronym \\ TEE $=$ transesophageal echocardiographic}

procedure cancelled, date last seen by nonsurgeon provider, reason for cancellation, date of rescheduled and performed cardiac surgery (when applicable), and death within 30 days of the cancelled procedure. For each patient, the interval from seeing a nonsurgeon provider to the scheduled surgery day was determined. For the purposes of classification, nurse practitioners and physician assistants working in the division of cardiac surgery were considered surgical providers.

The reasons for cancellation were categorized as medical, administrative or scheduling, patient cancellation, procedure no longer required, preincisional complication, or unknown. The category of medical cancelations included all noniatrogenic medical causes of cancellation. Administrative cancellations were those involving scheduling, operating room time, supplies, patient transport from an outside hospital, and insurance issues. Patient cancellations included those patients electing not to proceed with surgery on the day scheduled. Preincisional cancellations referred to circumstances in which cancellation occurred after the induction of anesthesia and included procedural, allergic, or anesthetic complications before the start of the operation, such as oropharyngeal bleeding during intubation or anaphylaxis.

Each cancellation was individually reviewed and was then classified as either foreseeable or nonforeseeable. Foreseeable cancellations were defined as preventable causes of same-day cancellation that a provider could have identified before the day of surgery from the available data and/or documented clinical symptoms. Nonforeseeable cancellations were those thought to not have been identifiable before the day of surgery. Indeterminate cancellations lacked sufficient documentation to determine a foreseeable or nonforeseeable cause. The classification of foreseeable status was made independently by 2 reviewers (M.M.S. and D.W.B.).

Statistical analysis consisted of determination of the mean, standard deviation, median, and range for the continuous variables and percentage quantification for the categorical variables. The foreseeable and nonforeseeable cancellations were analyzed in relation to whether the patient had seen a nonsurgeon provider within $15,30,45$, or 60 days of the planned operation using a 2 -sided $P$ value for differences in proportions using Fisher's exact test, determined using Statistical Analysis Systems, version 9.3 (SAS Institute, Cary, NC).

\section{RESULTS}

A total of 7081 cardiac surgical cases with cardiopulmonary bypass were performed within a 3-year period. During this period, 134 patients experienced 142 same-day cardiac surgery cancellations (Figure 1). Of these 134 patients, 6 had had 2 cancellations and 1 had had 3 cancellations of planned cardiac surgery. The overall same-day cancellation rate was $2.0 \%$ of the procedures performed (142 of 7081). The reasons for cancellation are listed in Table 1. Most $(51 \%)$ of the cancellations were for medical reasons; administrative or scheduling $(17 \%)$ and unknown reasons $(12 \%)$ were the next most commonly occurring causes.

The general findings pertaining to same-day cancellations are listed in Table 2. No discrepancies were noted between the 2 reviewers for the determination of foreseeable, nonforeseeable, and indeterminate cancellations. For 114 of the 142 patients who subsequently underwent surgery, the mean interval from cancellation to the actual cardiac operation was $12.2 \pm 22.7$ days (median, 1; range, $0-122$ ). The 0-day interval represented a patient whose procedure was cancelled intraoperatively, before the induction of anesthesia, owing to the need to perform an emergency operation for a different patient. The patient's procedure was subsequently rescheduled, and the patient underwent surgery later the same day. The patient with a 122-day interval from cancellation to surgery had developed intraoperative anaphylaxis shortly after anesthesia induction and had also experienced esophageal perforation from transesophageal echocardiographic (TEE) probe placement.

A total of 7 deaths occurred within 30 days of cancellation, of which 1 was preceded by a foreseeable cancellation. For all 7 patients who died, cancellation was related to medical reasons; 4 had not undergone surgery before death and 3 had subsequently undergone surgery before death. The single foreseeable cancellation preceding death involved 1 patient with untreated pneumonia. The pneumonia had been radiographically documented the day before surgery, and the patient was admitted for pneumonia and sepsis treatment and had died within 24 hours.

Of 142 cancellations, 7 had no record of a previous evaluation by a nonsurgeon provider. The mean interval from seeing a nonsurgeon provider to the planned operation was $8.6 \pm 16.2$ days (median, 1 ; range, $0-70$ ), with 0 days most commonly representing in-patients whose procedure was cancelled on the day of surgery. A greater percentage of patients experiencing foreseeable compared with nonforeseeable cancellations had not been seen by a nonsurgeon provider $15,30,45$, and 60 days before cardiac surgery (Figure 2). The data were not appropriate for statistical testing for trend owing to the small cell sizes.

Of the 142 cancelled surgical procedures, 28 were never subsequently performed. Of these 28 cancellations, 11 $(39 \%)$ occurred intraoperatively. The most common reason for cancellation involved the procedure no longer being required and/or TEE findings in 10 of $28(36 \%)$. The other reasons for cancellation involved 4 patients who elected to cancel, unknown reasons for 3, and other less common reasons for the remaining patients. The cancellation of procedures involving valve repair or replacement included 21 of $28(75 \%)$, removal of cardiac masses in $4(14 \%)$, coronary artery bypass grafting in $2(7 \%)$, and septal myectomy in $1(4 \%)$. Most $(82 \%)$ of these patients had seen a nonsurgeon provider within 45 days of their planned surgery.

\section{DISCUSSION}

The main findings of the present study were that same-day cancellations of cardiac surgery at our institution were very infrequent $(2 \%$ of all cardiac operations performed) and that foreseeable causes constituted a small fraction of those cancellations. Of all the cancelled 


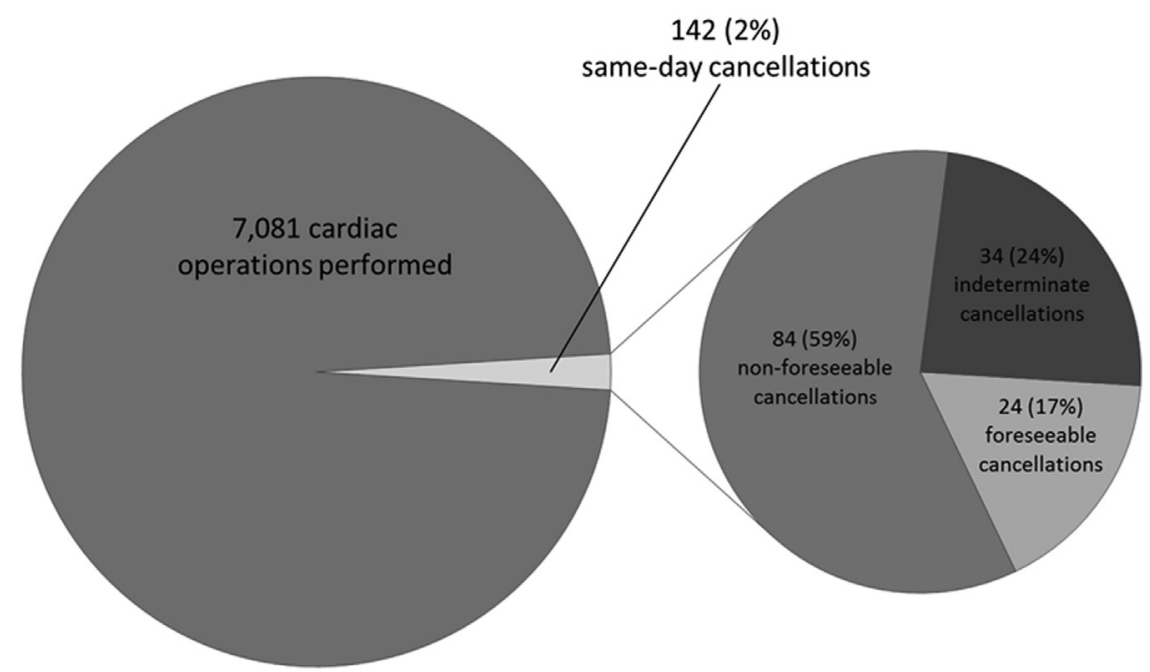

FIGURE 1. Quantification of cardiac surgical same-day cancellations in relation to total cardiac operations performed from 2010 to 2012.

operations, $20 \%$ were ultimately never performed, and patient deaths within 30 days of cancellation occurred in $5 \%$ of all cancelled cases. Finally, a more recent visit to a nonsurgeon provider did not affect foreseeable cancellations relative to nonforeseeable ones.

TABLE 1. Reasons for cancellation

\begin{tabular}{lc}
\hline \multicolumn{1}{c}{ Reason } & n (\%) \\
\hline Medical & $72 / 142(51)$ \\
Infection or fever & 25 \\
Other* & 21 \\
Acute kidney injury or dehydration & 4 \\
Volume overload or respiratory distress & 4 \\
Left atrial thrombus & 3 \\
Gastrointestinal bleeding & 3 \\
Neurologic symptoms & 2 \\
Preoperative coagulopathy or need for hematologic & 2 \\
$\quad$ consultation & \\
Required dental extractions before surgery & 2 \\
Rash & 2 \\
Required additional testing or workup & 2 \\
Hemodynamic instability following induction & 2 \\
Administrative or scheduling & $24 / 142(17)$ \\
Emergency case or unanticipated long preceding case & 14 \\
Scheduling problems & 4 \\
Supplies or prosthesis not available & 3 \\
Insurance issues & 2 \\
Late patient arrival from outside hospital transfer & 1 \\
Unknown & $17 / 142(12)$ \\
Procedure no longer required & $16 / 142(11)$ \\
Intraoperative TEE finding & 10 \\
Operation no longer believed to be required & 6 \\
Patient cancellation & $9 / 142(6)$ \\
Preincisional complication & $4 / 142(3)$ \\
Vascular access complication & 2 \\
Oropharyngeal bleeding in operating room before incision & 2 \\
\hline
\end{tabular}

$\overline{T E E}$, Transesophageal echocardiography. *Each individually represented $<1 \%$ of reasons for cancellation; 1 case of a patient not adhering to nothing by mouth guidelines was included in this group.
Same-day cancellation in surgical practice has costly financial implications. In 1 US-based study, revenue loss per cancelled case among a variety of surgical specialties was estimated to range from $\$ 850$ to $\$ 2025$, with the revenue loss for cardiac operations expected to be greater than that for many others. ${ }^{1}$ Although in many instances of cancellation, the next scheduled operation can be expedited to reallocate potentially idle resources and lost revenue, this might not be possible in all cases and will typically result in underusage of the operating rooms and staff. In addition, patients have described added anxiety and disappointment related to cancellation of cardiac surgery. ${ }^{2}$ Overall, previous studies have reported operative cancellation rates across all surgical specialties of $5 \%$ to $17.6 \%,{ }^{1,3-9}$ with specific cardiac surgical cancellation rates of $15 \%$ to $24 \%$. $^{1,3-5}$ Only 1 study from the Middle East specifically considered cancellation of cardiac surgery alone. They found a same-day cancellation rate of $15 \% .^{3}$ We found a much lower cardiac surgery same-day cancellation rate of $2.0 \%$. We attributed this relatively low rate of same-day cancellation largely to the institutional multidisciplinary approach to patient care; however, this could also have been related to our exclusion criteria (surgical procedures not requiring cardiopulmonary bypass; eg, sternal washout, extracorporeal membranous oxygenation weaning).

Our multidisciplinary model of patient care typically involves patients referred to a surgeon by a nonsurgical provider who supports the treatment of the patient in the preoperative period or direct referral to a surgeon from an outside institution, in which case the patient will often have been seen preoperatively in conjunction by a cardiologist or internist, if deemed necessary, to address nonoperative issues, confirm diagnoses, and establish postoperative medical follow-up care. Additionally, institutional resources and scheduling procedures have minimized the delays that can be associated with 
TABLE 2. General findings and demographic information related to 142 same-day cancellations of cardiac operations $(n=142)$

\begin{tabular}{|c|c|}
\hline Variable & Value \\
\hline \multicolumn{2}{|l|}{ Age at cancellation $(\mathrm{y})$} \\
\hline Mean \pm SD & $68.3 \pm 13.9$ \\
\hline Median & 71.1 \\
\hline Range & $18-89$ \\
\hline Male gender & $94(66.2)$ \\
\hline \multicolumn{2}{|l|}{ Cardiac surgery eventually performed } \\
\hline Yes & $114(80)$ \\
\hline No & $28(20)$ \\
\hline \multicolumn{2}{|c|}{ Interval from cancellation to operation (d) } \\
\hline Mean \pm SD & $12.2 \pm 22.7$ \\
\hline Median & 3.0 \\
\hline Range & $0-122$ \\
\hline Death within $30 \mathrm{~d}$ of cancellation & $7(5)$ \\
\hline \multicolumn{2}{|l|}{$\begin{array}{l}\text { Interval from planned surgery to seeing } \\
\text { nonsurgeon provider* (d) }\end{array}$} \\
\hline Mean \pm SD & $8.6 \pm 16.2$ \\
\hline Median & 1 \\
\hline Range & $0-70$ \\
\hline \multicolumn{2}{|l|}{ Cancellation location } \\
\hline Preoperative & $117(82)$ \\
\hline Intraoperative & $25(18)$ \\
\hline \multicolumn{2}{|c|}{ Reason for cancellation deemed foreseeable } \\
\hline Yes & $24(17)$ \\
\hline Medical & $17 / 24(71)$ \\
\hline Procedure no longer required & $4 / 24(17)$ \\
\hline Administrative or scheduling & $3 / 24(12)$ \\
\hline No & $84(59)$ \\
\hline Indeterminate & $34(24)$ \\
\hline \multicolumn{2}{|l|}{ Surgical procedure cancelled } \\
\hline Valve $\dagger$ & $57(40)$ \\
\hline CABG & $29(20)$ \\
\hline $\mathrm{CABG}$ and valve $\dagger$ & $19(13)$ \\
\hline Percutaneous valve replacement & $11(8)$ \\
\hline VAD with or without valve $\dagger$ & $11(8)$ \\
\hline Cardiac mass or thrombus excision & $5(4)$ \\
\hline Other $\ddagger$ & $4(3)$ \\
\hline Septal myectomy & $3(2)$ \\
\hline Aortoplasty & $3(2)$ \\
\hline
\end{tabular}

Data presented as $\mathrm{n}(\%)$, unless otherwise noted. SD, Standard deviation; $C A B G$, coronary artery bypass grafting; $V A D$, ventricular assist device. *Interval from planned surgery to seeing a nonsurgeon provider was known for 135 of 142 patients. $\dagger$ Included all valve replacement or repairs performed by way of median sternotomy. $\ddagger$ Each individually represented $<1 \%$ of all procedures cancelled.

preoperative assessments and diagnostic testing to scheduling of the operative date. Nearly all cardiac surgery patients at our institution will be seen by a cardiac surgery nurse practitioner or physician assistant on the business day before surgery, and all patients will be seen within 30 days of the scheduled surgery. This allows for cancellation or postponement before the day of surgery, if necessary, and might explain both our relatively low rate of same-day cancellation and our success at minimizing foreseeable cancellations. From the present study, we were unable to determine whether the operational costs of our care model (preoperative consultations and the cardiac surgical outpatient appointment the day before the scheduled operation) could account for our low cancellation rate. However, we believe that these preoperative consultations have been paramount to achieving the reported results and overall patient satisfaction at our institution.

We are unaware of guidelines or recommendations describing the optimal timing for the medical evaluation by a nonsurgeon provider before cardiac surgery. One might theorize that seeing a nonsurgical provider closer to the planned surgery might reduce foreseeable cancellations. However, we did not find this to be true in the present series. Overall, most $(51 \%)$ of the cancelled cases at our institution had been cancelled for medical reasons, which were largely nonforeseeable. A greater portion of patients with a foreseeable cancellation (vs a nonforeseeable cancellation) had not seen a nonsurgeon provider; however, these differences were not statistically significant. However, it is possible that seeing a nonsurgeon provider before planned surgery could be associated with fewer foreseeable cancellations in a larger sample size, if the interval between the evaluation and planned operation were greater or in practices with a different referral model than ours.

Cancellation of cardiac surgery without subsequent operation represented $20 \%$ of our cancellations. Most of those not undergoing a future operation involved patients electing to cancel, intraoperative echocardiographic findings nullifying the initial operative indication (eg, cardiac mass no longer present), or unknown reasons. Additionally, it is possible that some of the remaining cancellations without a subsequent surgery might have involved cases best deemed inoperable during the initial evaluation. However, we were unable to definitely make this determination given the retrospective nature of the present study. Being a large tertiary referral center, patients will often be scheduled for surgery with the expectation that additional testing will occur around the time of, or even on the same day of, a scheduled operation. This late testing has the potential to negate the indication for cardiac surgery. Additionally, a large proportion of cancellations without a subsequent operation were cancelled intraoperatively $(39 \%$ vs $18 \%$ overall), most commonly because of intraoperative TEE findings. This observation reaffirms a benefit of intraoperative transesophageal echocardiography, because an alteration in the planned operation from the TEE findings has been previously described. ${ }^{10}$ Finally, each of the 7 deaths after cancellation involved medical reasons for cancellation, likely reflecting the high acuity and comorbid conditions increasingly present in cardiac surgery patients.

The limitations of the present study included all those inherit to a retrospective series, such as medical record omissions and that the reasons for cancellation might not have been discernible on retrospective review. However, 


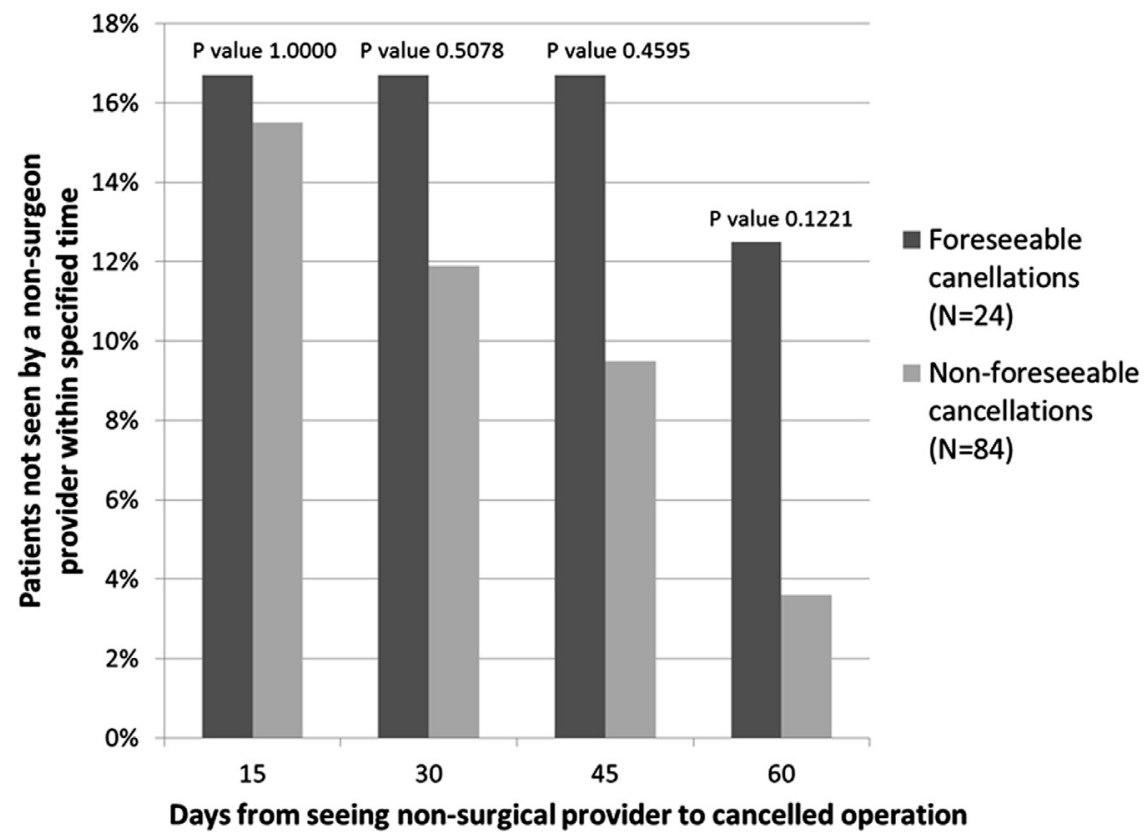

FIGURE 2. Percentage of patients experiencing foreseeable and nonforeseeable cancellations who were not seen by a nonsurgeon provider before the planned operation.

only a few of the cancellations ( $12 \%$ ) occurred for reasons that were indeterminate. Given that many patients will present to our tertiary referral center specifically for perioperative care and then receive follow-up care elsewhere, we were unable to determine the patient satisfaction-related implications of cancellation in our retrospective series. Future studies are needed to elucidate this important aspect of operative cancellation.

\section{CONCLUSIONS}

Same-day cancellations in cardiac surgery occurred infrequently at our institution and at a rate much lower than previously reported. The foreseeable causes for cancellation comprised only a few of our cancellations, which we attributed to the successful preoperative multidisciplinary approach to patient care and an outpatient visit the business day before surgery by a cardiac surgery "midlevel" provider. Although different institutions presumably experience widely varying cancellation rates, thinking of cancellations as inevitable within busy surgical practices should be avoided and instead viewed as opportunities for practice improvement.

\section{References}

1. Argo JL, Vick CC, Graham LA, Itani KM, Bishop MJ, Hawn MT Elective surgical case cancellation in the Veterans Health Administration system: identifying areas for improvement. Am J Surg. 2009;198:600-6.

2. Ivarsson B, Larsson S, Sjoberg T. Postponed or cancelled heart operations from the patient's perspective. J Nurs Manag. 2004;12:28-36.

3. Farasatkish R, Aghdaii N, Azarfarin R, Yazdanian F. Can preoperative anesthesia consultation clinic help to reduce operating room cancellation rate of cardiac surgery on the day of surgery? Middle East J Anesthesiol. 2009;20:93-6.

4. Schofield WN, Rubin GL, Piza M, Lai YY, Sindhusake D, Fearnside MR, et al. Cancellation of operations on the day of intended surgery at a major Australian referral hospital. Med J Aust. 2005;182:612-5.

5. Hand R, Levin P, Stanziola A. The causes of cancelled elective surgery. Qual Assur Util Rev. 1990;5:2-6.

6. Kumar R, Gandhi R. Reasons for cancellation of operation on the day of intended surgery in a multidisciplinary 500 bedded hospital. J Anaesthesiol Clin Pharmacol. 2012;28:66-9.

7. Leslie RJ, Beiko D, Van Vlymen J, Siemens DR. Day of surgery cancellation rates in urology: identification of modifiable factors. Can Urol Assoc J. 2012; 10:1-8.

8. Sanjay P, Dodds A, Miller E, Arumugam PJ, Woodward A. Cancelled elective operations: an observational study from a district general hospital. J Health Organ Manag. 2007;21:54-8.

9. Schuster M, Neumann C, Neumann K, Braun J, Geldner G, Martin J, et al. The effect of hospital size and surgical service on case cancellation in elective surgery: results from a prospective multicenter study. Anesth Analg. 2011;113: 578-85.

10. Click RL, Abel MD, Schaff HV. Intraoperative transesophageal echocardiography: 5-year prospective review of impact on surgical management. Mayo Clin Proc. 2000;75:241-7. 